\title{
Diacronie
}

Studi di Storia Contemporanea

$\mathrm{N}^{\circ} 28,4 \mid 2016$

La voce del silenzio

\section{Ricardo Martín de la Guardia, El europeísmo. Un reto permanente para España}

\section{Matteo Tomasoni}

\section{Q OpenEdition \\ 1 Journals}

Edizione digitale

URL: https://journals.openedition.org/diacronie/4603

DOI: $10.4000 /$ diacronie.4603

ISSN: 2038-0925

Editore

Association culturelle Diacronie

Notizia bibliografica digitale

Matteo Tomasoni, «Ricardo Martín de la Guardia, El europeísmo. Un reto permanente para España»,

Diacronie [Online], № 28, 4 | 2016, online dal 29 décembre 2016, consultato il 21 septembre 2021. URL: http://journals.openedition.org/diacronie/4603; DOI: https://doi.org/10.4000/diacronie.4603 


\title{
Diacronie
}

N. 28 | 4|2016 La voce del silenzio: intelligence, spionaggio e conflitto nel XX secolo

\section{4/}

\section{PANORAMICA: Spagna 2015}

\author{
Antonio César MORENO CANTANO, Matteo TOMASONI *
}

GONZÁleZ CALLEJA, Eduardo, COBO ROMERO, Francisco, MARTíneZ RUS, Ana, SÁNCHEZ PÉREZ, Francisco, La Segunda República española, Barcelona, Pasado \& Presente, 2015, 1373 pp.

a cura di Matteo TOMASONI

Nel corso degli ultimi anni il dibattito sulla Seconda Repubblica spagnola si è notevolmente ampliato, stimolando non poche riflessioni poi convertitesi in importanti spunti di ricerca. Non è quindi un caso che ancora oggi il panorama storiografico spagnolo sia contraddistinto da studi che promuovono l'approfondimento e la comprensione di un periodo definito spesso come "travagliato" e "conflittuale", senza però tenere in considerazione (o limitandosi ad indicarlo solo in alcuni casi) anche l'enorme peso che tale periodo ebbe sulla configurazione della società e della cultura spagnola dell'epoca. È proprio a partire da queste basi che un rinomato gruppo di ricercatori spagnoli è voluto partire; così come viene specificato nel prologo, l'opera in questione «non vuol infatti trasformarsi in una visione fatalista o masochista della storia repubblicana», dal momento che pretende di far luce «senza alcun pregiudizio» su di un periodo storico considerato ancor oggi controverso. Il dibattito si sviluppa in più direzioni, sebbene la tesi di partenza sia proprio quella delle "visioni contrapposte" dove sussistono diverse opinioni sulle origini, lo sviluppo e le conseguenze di un regime repubblicano durato appena pochi anni (1931-1939). Tendenzialmente vi sono due linee interpretative che caratterizzano il regime repubblicano: da una parte chi ha sempre difeso il fatto che questo sistema politico sia il risultato di un processo di modernizzazione sorto come risposta alla grave crisi nazionale di fine Ottocento, dall'altra chi ha invece addossato a questo sistema politico la responsabilità per essere divenuto il momento culminante di un'epoca di decadenza iniziata con la Restauración 
e quindi conclusasi - così come sostenuto da alcuni storici di epoca franchista - con lo sviluppo di un inevitabile conflitto civile che aprì le porte ad una dittatura durata quarant'anni. Durante quest'ultimo periodo il regime di Francisco Franco promosse un’immagine falsata della Repubblica, spesso accusata e additata come unica responsabile - si tengano presenti i chiari connotati filo-fascisti del regime franchista dei problemi sociali, del processo di sovietizzazione della nazione, ma anche, questione ben più importante nella configurazione dello Stato autoritario di Franco, dell'imprudente svolta anticlericale voluta dalla democrazia repubblicana. Quella che i franchisti sostennero essere stata una pericolosa rivoluzione in ragione della quale giustificarono la loro ribellione (alcune destre politiche la chiamarono 'controrivoluzione'), fu difesa da altri in termini diametralmente opposti, con l'intenzione di segnalare come tale sistema politico fosse piuttosto un regime volutamente popolare basato sul cambiamento, la modernizzazione, la conquista dei diritti sociali, ma anche sul riformismo. L'aspettativa originata da questo processo di rinnovamento costituì forse una delle principali carte che la Repubblica poté giocare durante la sua esistenza, specialmente durante il primo biennio (1931-1933), ma le pessime condizioni economiche del momento, i limiti di questa trasformazione, la scarsa gestione delle opere di riforma e la progressiva radicalizzazione politica della società, non fecero altro che frenare proprio la modernizzazione conclamata sin dalla proclamazione del nuovo regime. Con il passar del tempo le tesi più varie si sono accumulate nel tentativo di dare una spiegazione a questo apparente fallimento politico, nonostante i connotati di rigidità del regime instauratosi successivamente - il franchismo - che non fece nulla per salvaguardare l'immagine della Repubblica. Oggi tocca quindi agli storici far luce e mantenere aperto il dibattito su questo periodo storico: non si tratta solo di riflettere su quelle che furono le aspettative del regime democratico e quali gli effetti che esso produsse sulla popolazione spagnola, ma anche sui sentimenti, sulle passioni e sulla speranza o sulla sfiducia (a seconda del caso) che tale sistema generò. Ed è proprio questa riflessione la base di quella che gli autori di questa pubblicazione chiamano il «paradosso dell'afflizione», riferendosi alla Repubblica come a un'occasione persa o a un progetto incompiuto. Ma tale affermazione, oltre all'evidenza dei fatti, permette anche di meditare su quello che molti hanno definito esser stato il naturale epilogo di una Repubblica con queste caratteristiche e cioè il conflitto civile stesso. Eppure, tale interpretazione non può essere considerata un dato di fatto: il regime repubblicano non è giudicabile come unico responsabile di un conflitto di quelle di quelle dimensioni, ma piuttosto un attore che si vide coinvolto - forse a causa dei suoi stessi errori - in un processo vertiginoso 
che lo coinvolse nel più violento degli scenari possibili: la guerra civile. Questo libro si pone come una riflessione in grado di ampliare, ripensare e riproporre i grandi progetti sociali, politici, economici, organizzativi, che la Repubblica cercò di mettere in atto durante la sua breve vita: dal progetto democratico, basato su di una delle Costituzioni più innovative dell'epoca, alla proposta riformista, dalla modernizzazione della società ad una maggior libertà politica e sociale, dallo stimolo culturale ed educativo alla parità tra i sessi, dallo stato laico alla libertà religiosa. Non uno ma numerosi spunti di riflessione che, ad ottant'anni dall'inizio della guerra civile, è doveroso riprendere in considerazione.

\section{NÚÑEZ SEIXAS, Xosé Manoel, Las utopías pendientes. Una breve historia del mundo desde 1945, Barcelona, Crítica, 2015, 383 pp. a cura di Matteo TOMASONI}

L’ultima pubblicazione del professor Xosé M. Núñez Seixas si presenta come un testo imprescindibile per chi - sia per gli specialisti, sia per semplice appassionati intenda riflettere sull'appassionante, intensa e spesso complessa storia degli ultimi settant'anni. Attraverso uno studio che impone la prospettiva transnazionale in aree tematiche come la storia di genere, la memoria collettiva, le identità nazionali, ma anche - questione spesso dimenticata - l'accrescimento della preoccupante questione ambientale, l'autore ci accompagna in un viaggio il cui intento è quello di stimolare la riflessione. L'epoca delle «utopie in sospeso» (questa la traduzione letterale del titolo) non è quindi altro che la volontà di riflettere e far riflettere su di un ampio periodo storico che ha dovuto fare i conti con il suo immediato passato (quello dei grandi conflitti mondiali), cercando di proporre possibili alternative politiche, sociali, ma anche economiche, basate sulle esperienze maturate sino a quel momento. Il mondo uscito dall'inferno del 1945 rimase a lungo bipolare, ma è entrato in un processo di multipolarizzazione, oggi definito con il termine globalizzazione, proprio ad indicare il fenomeno di diffusione uniforme e convergente delle pratiche sociali, politiche ed economiche sviluppatosi alla fine del XX secolo. Il libro dà molto spazio a questa riflessione, suggerendo le cause che hanno portato a questa situazione, tenendo in considerazioni i fatti e le conseguenze che causò soprattutto la fine della Guerra Fredda. Non è un caso che Núñez Seixas parta proprio dall'Europa occidentale in piena metamorfosi tra gli anni Sessanta e Settanta, per poi analizzare gli effetti provocati dalla caduta del blocco comunista, il ruolo degli Stati Uniti nella politica internazionale specialmente nel periodo successivo alla guerra del Vietnam, ma anche l'emergere di 
società sino ad allora considerate complementari come la Cina popolare o le "Tigri asiatiche"; tutto ciò senza ovviamente dimenticare la decolonizzazione dell'Africa o le dittature militari dell'America Latina. Due capitoli centrali del libro sono incentrati su temi con un peso del tutto particolare nella fisionomia del secondo dopoguerra. In uno di essi viene ricordata la difficile convivenza tra l'imperante necessità di lasciarsi alle spalle il dramma della guerra e, al contempo, la doverosa memoria dell'Olocausto (una sezione è dedicata proprio al dibattito interno alla RFT, ma anche ad altri casi in cui l'esistenza di regimi totalitari portò a gravissimi casi di genocidio); non meno importante è capire le dinamiche attraverso cui l'Occidente tentò di plasmare l'utopia di una pace duratura a livello mondiale o lo sforzo - tutto sovietico - di generare consenso politico attraverso la creazione di una memoria collettiva attraverso la causa antifascista o l'esaltazione della Grande guerra patriottica. Ci immergiamo poi nell'analisi del nazionalismo successivo alla Seconda guerra mondiale, cercando di capire quali furono i principali processi di sviluppo di nuove e vecchie identità, quale sia il significato di "nuovo nazionalismo", ma anche quali interpretazioni vadano consolidandosi intorno all'idea di nazione, così come dimostra l'odierna esistenza di un forte sentimento identitario in determinate regioni come dimostra la frammentazione politica europea post ' 89 . Un dibattito che però ha dovuto fare i conti anche con il progetto unitario europeo, che ha posto in evidenza - sottolinea Seixas - la creazione di «un'Europa delle regioni e dei popoli come alternativa all'Europa degli Stati nazionali». I rimanenti capitoli del libro sono dedicati a temi di attualità come l'emancipazione femminile, il problema ambientale e del surriscaldamento globale, ma anche degli effetti provocati dalla globalizzazione. È qui che si celano le nuove incognite sociali e politiche del XXI secolo: l'impossibilità di creare egemonie mondiali come quella statunitense, la crescita e il maggior protagonismo del mondo islamico, le grandi sfide democratiche iniziate nei paesi tradizionalmente autoritari o semi-autoritari, la nuova liberalizzazione economica e la crisi del capitalismo. Un sistema-mondo che quindi, conclude il professor Núñez Seixas, deve fare i conti con le molte «utopie ancora in sospeso»: a quelle di ieri bisogna sommare quelle di oggi.

\section{DE LA GUARDIA, Ricardo Martín, El europeísmo. Un reto permanente para España, Madrid, Cátedra, 2015, 344 pp.}

a cura di Matteo TOMASONI

Il celebre filosofo José Ortega y Gasset scrisse alla fine degli anni Venti del XX secolo, che «fu il realismo storico ad insegnarmi che l'unità della società europea non è 
un "ideale", ma un fatto di antica quotidianità»1. La creazione dell'ipotetica «unione degli Stati europei» fu pronosticata dall'intellettuale spagnolo proprio con l'idea che essa sarebbe stata una logica forma di sviluppo sociale e politica del vecchio continente, sebbene non esente da incognite e dubbi sulla sua reale fattibilità. Si trattava quindi, asseriva Ortega y Gasset, di pensare alla futura Europa unita come ad un continentenazione o piuttosto ad un insieme multinazionale unito semplicemente da un sentimento comune? Ma che tipo di sentimento? E quali dovevano essere le sue caratteristiche, le sue responsabilità e quale - oggetto di molti dibattiti - la sua forma di governo? A più di ottant'anni di distanza da quelle riflessioni, ci ritroviamo oggi in un'Europa che si definisce "unita", "solida" ed allo stesso tempo impegnata in una "politica comune". Questo, stando alle dichiarazioni che giungono da Bruxelles, è quello che ci viene ripetuto ogni giorno sebbene i dubbi siano ancora molti: in che modo l'Unione Europea (UE) si è realmente consolidata nelle strutture politicoamministrative dei paesi che la compongono? Quanto partecipiamo (come cittadini) alle decisioni politiche, all'economia o all'organizzazione della UE? Ed ancora: la UE può e deve essere considerata una soluzione ai problemi del vecchio continente o è diventata oggi un'ulteriore preoccupazione per gli Europei? Tutte queste domande sono state oggetto di un'interessante riflessione portata avanti dal professor Ricardo Martín de la Guardia, che ha cercato di dare una risposta a tali quesiti attraverso uno studio sulla partecipazione della Spagna al processo politico europeo degli ultimi decenni. Il suo libro non vuole però essere una semplice analisi della situazione venutasi a creare durante la fase di consolidamento e integrazione della Spagna alla UE, ma piuttosto cerca di essere un ragionamento sulle fasi che hanno caratterizzato il graduale, lento e talvolta complicato - spesso conflittuale - avvicinamento del paese iberico alla quotidianità di Bruxelles. Ma Martín de la Guardia avvia la sua riflessione da molto prima di questo passaggio storico: sottolinea come il concetto di Europa penetrò nell'animo degli Spagnoli a partire dai tempi dell'Illuminismo, quando il vecchio continente era concepito come il faro dell'umanità e il simbolo del progresso civile. Lo sviluppo scientifico, l'universalizzazione della cultura, le riforme sociali, politiche ed economiche, ma anche una nuova percezione della società, contribuirono a creare le basi di un nuovo avvicinamento tra una Spagna che cercava di abbandonare il suo status di "nazione periferica" e un'Europa sempre più protagonista della politica mondiale. Le conseguenze dall'ascesa della Francia napoleonica prima e dopo il Congresso di Vienna, provocarono un certo stallo nei rapporti ispano-europei, che si diradarono nel XIX secolo, nonostante il riemergere di idee pro-europeiste come quelle

${ }^{1}$ ORTEGA Y GASSET, José, La Rebelión de las masas, Madrid, Tecnos, 2009, pp. 83-84. 
avanzate da importanti pensatori iberici come Jaime Balmes o Donoso Cortés. L'interesse di questi autori fu quello di riflettere intorno alle relazioni sociali e politiche che la Spagna doveva mantenere con il resto dell'Europa, ma soffermarsi anche sul tipo di rapporto che sarebbe dovuto maturare. Sino all'inizio della Grande guerra - che cancellò definitivamente quel fragile equilibrio fissato proprio a Vienna nel 1815 - il concetto di Europa in Spagna rimase prevalentemente legato alla sfera culturale anche se la grande crisi di fine secolo provocò proprio un ritorno al dibattito europeista grazie al contributo intellettuale voluto dalla Generación del '98. L'effetto provocato da questa riflessione si protrasse durante i primi decenni del XX secolo e influenzò in grande misura la creazione di un movimento pro-europeista che in parte venne difeso anche dal governo democratico sorto nel 1931. Fu un'epoca di intenso dibattito sociale e politico in cui si trovava coinvolta tutta l'Europa, che ebbe però risultati assai diversi: mentre il fascismo scomparve in buona parte del continente, in Spagna il regime di Francisco Franco si consolidò e perdurò per quarant'anni, raffreddando notevolmente le relazioni che con difficoltà avevano riavvicinato la Spagna all'Europa. Una situazione che cambiò tra la fine degli anni Sessanta e l'inizio degli anni Ottanta, quando la Comunità Europea iniziò a giocare un ruolo sempre maggiore nella politica estera della Spagna. La Transizione democratica, il riassetto sociale e politico del paese iberico ormai in una fase "post-franchista" e l'evidente accelerazione europeista imposta dal governo di Adolfo Suárez, diedero vita al processo di integrazione del paese iberico nell'Unione, suggellata anche dall'impegno politico preso dal socialista Felipe González. Un progetto che ebbe un notevole successo ed appoggio iniziale ma che, recentemente, ha iniziato ad intaccare la società iberica specialmente a causa degli effetti provocati dalla recente crisi economica (e dall'adozione di politica di austerità) o dal "potere decisionale" di Bruxelles; un dialogo considerato da molti come unidirezionale dove è venuto a meno il confronto diretto e quindi la stabilità dello stesso accordo di collaborazione. 


\title{
CERVERA GIL, Javier, Contra el enemigo de la República... desde la Ley.
} Detener, juzgar y encarcelar en la guerra, Madrid, Biblioteca Nueva, 2015, 238 pp.

\author{
a cura di Antonio César MORENO CANTANO \\ traduzione di Matteo TOMASONI
}

Le visioni revisionistiche neofranchiste hanno spesso rappresentato la Spagna repubblicana - e specificamente quella del '36 - come uno Stato ormai in balia di gruppi rivoluzionari incapaci di esercitare e imporre il proprio potere rispetto ad un fenomeno politico - il franchismo - sorto dopo il fallimento del colpo di stato del 18 luglio. Ricorrendo a fonti documentarie provenienti da numerosi archivi nazionali, il Professor Javier Cervera della Universidad Francisco de Vitoria di Madrid ha cercato di opporsi a questa tesi proponendo un dettagliato studio nel quale si vuole analizzare a fondo lo sforzo del Governo repubblicano contro l'abuso della violenza politica. Non più, quindi, una giustizia sommaria esercitata dai diversi elementi politici fedeli alla repubblica, ma l'instaurazione di un sistema di controllo giudiziario basato su tre fasi: detenzione, processo e condanna. La "nuova repubblica" sorta all'interno del processo rivoluzionario doveva essere - così come segnala l'autore - coerente con i tempi di guerra, ma soprattutto doveva normalizzare la persecuzione politica nella retrovie. I dirigenti repubblicani cercarono - diversamente dalla prassi che venne poi adottata all'interno del bando franchista - di porre fine a questa violenza (specialmente a quella anarchica e comunista), per ragioni di coscienza o come semplice rifiuto della pratica della 'giustizia sommaria', ma anche a causa della ripercussioni diplomatiche che si sarebbero potute ritorcere in qualsiasi momento contro la causa repubblicana. Anche se in un primo momento la propaganda riuscì a migliorare l'immagine della Repubblica all'estero, durante buona parte della Guerra Civile la Spagna democratica fu spesso additata come un regime avverso alla Chiesa cattolica (dibattito vivo in paesi come Francia, Stati Uniti o Regno Unito); un'accusa che indebolì fortemente la causa repubblicana a livello internazionale. La presenza di una giustizia legata alla pratica della violenza politica consistette quindi, inevitabilmente, in un maggior controllo della retrovie, ma anche in una riorganizzazione del sistema giudiziario e degli organismi vicini alle forze dell'ordine, così come del proprio sistema carcerario. Questo criterio diede inizio ad una 'nuova fase' dell'amministrazione repubblicana nel 1937, molto differente - anche a causa delle circostanze belliche del momento - a quella dell'anno 1931. Misure di controllo come il Consejo Nacional de Seguridad, la proibizione per 
ogni singolo cittadino, partito politico o organo sindacale o comité di possedere armi, l'organizzazione di tribunali popolari o di Jurados de Guardia y de Urgencia o, ancora, la realizzazione di campi di lavoro forzato: tutti questi elementi furono, ognuno con le sue peculiarità, un esempio della volontà tutta repubblicana di favorire un cambiamento reale nella nazione. Eppure il regime repubblicano non riuscì mai a controllare questo sistema così come aveva previsto: le retrovie erano ancora (e lo furono sino alla conclusione del conflitto) un luogo di persecuzione ideologica e politica, divenendo - paradossalmente - una delle cause dell'imminente sconfitta. Ed è proprio su questo punto che lo studio in questione cerca di far luce, cercando di mettere in evidenza gli sforzi delle autorità repubblicane nel combattere un nemico onnipresente, senza smarrire l'obiettivo di controllare e regolare il sistema giudiziario.

\section{GóMEZ OCHOA, Fidel, GOÑI PÉREZ, José M., MACÍAS FERNÁNDEZ,} Daniel (Eds.), La guerra. Retórica y propaganda (186o-1970), Madrid, Biblioteca Nueva, 2015, 254 pp.

a cura di Antonio César MORENO CANTANO

traduzione di Matteo TOMASONI

In questi ultimi anni stiamo assistendo ad un importante recupero - concettuale ma anche metodologico - degli studi di storia militare, almeno per quanto riguarda il caso spagnolo. Nuovi obiettivi ma anche nuove visioni ed interpretazioni dei fenomeni legati ai concetti di violenza, guerra e istituzioni militari sono sorti proprio con lo scopo di rigenerare questo campo di studi. L'origine di questa riscoperta degli studi dedicati al mondo bellico sorse concretamente presso l'Universidad de Cantabria, intorno all'anno 2000, con la creazione del CIHBE (Coloquio Internacional de Historia Bélica). Durante il secondo congresso Los lenguajes de la guerra..., il dibattito ruotò intorno alla questione della complessità iconico-discorsiva della guerra, ma anche alla difficoltà di comunicazione del fenomeno bellico, motivo per il quale fu necessario ricorrere ad un approccio multidisciplinare sostenuto dalla psicologia, dalle arti, dalle scienze politiche, dalla sociologia ed anche dall'estetica. L'incontro fu un grande successo all'interno del quale vennero presentate proposte scientifiche provenienti da numerosi paesi; $\mathrm{i}$ contributi più innovativi e di maggior importanza sono stati inseriti nell'opera in questione. Non è un caso quindi che si sviluppi su di una riflessione che approfondisce il ruolo della retorica e della propaganda di guerra, rivendicando - in linea con l'eccellente lavoro di Peter Burke, Eyewitnessing. The User of Images as Historical 
Evidence del 2001 - il fenomeno della propaganda visiva (quello che oggi conosciamo come manifesti, comunicati, volantini, ...). All'interno di quest'opera si propone una dettagliata analisi dei principali studi esistenti sulla propaganda bellica (Arthur Ponsoby, Harold D. Lasswell, Philip Taylor o Pizarroso Quintero), ma si ricorda anche lo scarso interesse (durato sino agli anni Novanta del secolo scorso) che si è avuto nei confronti di questa disciplina, almeno fra gli storici spagnoli. L'espressione storia militare è considerata antiquata essendo oggi più consono l'uso di concetti come historia de lo bélico (questa definizione viene rivendicata, per esempio, dalla «Revista Universitaria de Historia Militar», che insiste su aspetti come l'esperienza del combattente, la prospettiva di genere durante la guerra o il punto di vista del civile non solo come soggetto passivo, ma attivo all'interno del conflitto...). Dei dodici capitoli del libro, la metà si sviluppano proprio intorno al caso spagnolo. Temi come la guerra del Rif (María Gajate Bajo), la propaganda tedesca nella penisola iberica durante la Seconda guerra mondiale (Javier Ponte Marrero y Guillermo J. Pérez Casanovas), la fotografia e il cinema durante la guerra civile (Ángel Mato Díaz y Luis Veres), i manifesti di propaganda dei soldati repubblicani spagnoli durante la Seconda guerra mondiale (Pierre-Paul Gregorio y Adolfo Cueto Rodríguez) trovano spazio in queste pagine. Inoltre vengono presi in esame altri ambiti geografici, che vanno dal Portogallo alla Nuova Zelanda. Ultima, ma non meno importante, la riflessione sulla condizione fisica del soldato dalla Guerra di secessione americana fino alla Guerra del Vietnam. Su quest'ultimo aspetto la storica Joanna Bourke propone una riflessione tesa a valutare le modalità con cui sono cambiati i metodi di trasmissione della narrazione bellica attraverso i secoli XIX e XX. Da quello che viene definito come 'stoicismo' dei primi tempi si è passati ad una maggior sensibilità nei confronti dell'esperienza o delle condizioni psicologiche del soldato. Vengono qui ricostruite le diverse percezioni della guerra attraverso i rapporti sanitari, i diari dei combattenti, le testimonianze dell'epoca, che forniscono una visione globale del conflitto. Una ricerca che riunisce tutti quei fattori che si considerano imprescindibili per scrivere la nuova storia bellica: dall'interdisciplinarietà alle fonti storiche alternative, dalle nuove tematiche di studio agli effetti provocati dal conflitto. L'opera propone, infine, un'abbondante quantità di materiale grafico che logicamente aiuta il lettore ad approfondire gli sviluppi di questa ricerca; un lavoro che indubbiamente aspira ad essere un importante riferimento in quest'ambito storiografico. 
CAÑELAS MAS, Antonio, Alfredo Sánchez Bella. Un embajador entre las América y Europa. Diplomacia y política informativa en la España de Franco (1936-1973), Gijón, Ediciones Trea, 2015, 441 pp.

a cura di Antonio César MORENO CANTANO

traduzione di Matteo TOMASONI

Lo storico Antonio Cañellas ha dedicato una parte considerevole dei suoi studi al regime franchista e in particolare ad alcuni dei suoi principali attori politici. Tutto ebbe inizio con la sua tesi dottorale, dedicata a Laureano López Rodó, Ministro degli Esteri in uno degli ultimi governi di Franco, per poi approfondire la figura del cattedratico Vicente Rodríguez Casado principale artefice della Escuela de Estudios Hispanoamericanos. Grazie a queste e molte altre esperienze, Cañellas ha potuto dar vita ad un progetto che intende mettere in luce l'esperienza culturale del franchismo, le cui direttrici naturali sono state sia il vecchio continente che l'America Latina. Da questa riflessione è nata quindi l'opera in questione, la cui attenzione si è concentrata sull'operato dell'ambasciatore Alfredo Sánchez Bella ed il suo lavoro all'interno del Ministero degli Esteri e dell'Informazione e del Turismo (presieduti in quel momento da Alberto Martín Artajo e Fernando María Castiella). Sebbene l'opera dimostri un rigore scientifico impeccabile, caratteristico dell'autore, essa mantiene intatta anche la sua volontà divulgativa, creando quindi le basi di un lavoro destinato ad essere un valido esempio in quest'ambito di ricerca. Non bisogna però dimenticare che il professor Cañellas ha avuto anche la possibilità di accedere a numerosi fondi archivistici inediti, i quali sono stati fondamentali nel lavoro di ricostruzione potremmo dire quasi "chirurgico" - della traiettoria politica e biografica di Sánchez Bella. Oltre agli scritti personali, troviamo importanti documenti legati alle figure di López Rodó e Florentino Pérez Embid, ma anche altro materiale proveniente dall'Archivo General de la Administración, archivi stranieri (specialmente italiani) come la Fondazione Luigi Sturzo o i fondi della segreteria politica della Democrazia Cristiana. Il libro si sviluppa in sei capitoli che vanno dall'età giovanile a quella universitaria, che trattano il periodo in cui occupò la carica di direttore dell'Instituto de Cultura Hispánica, poi il ruolo di ambasciatore nella Repubblica Dominicana, in Colombia e in Italia, fino all'epoca in cui ricoprì il ruolo di Ministro dell'Informazione e del Turismo (1969-1973). Così come altri dirigenti del franchismo, specialmente quelli vincolati al governo tecnocratico e le personalità dell'Acción Católica, Bello si sforzò nel trovare il modo di prolungare l'azione politica del franchismo, affinché tale regime potesse sopravvivere alla scomparsa del Generalísimo Francisco Franco. Sánchez Bello 
fu indubbiamente l'ideologo dell'Estado presidencial coronado, ovvero una forma di governo il cui sviluppo avrebbe mantenuto in piedi - nonostante la promessa di una vaga apertura politica - il sistema corporativo, consolidando la struttura gerarchica di uno Stato risolutamente cattolico, presidenziale e monarchico; tutt'altro, quindi, che democratico. Come direttore dell'Instituto de Cultura Hispánica, Sánchez Bella era convinto del fatto che se i paesi ispanoamericani avessero recuperato il loro sentimento cattolico, avrebbero conosciuto un'importante rigenerazione sociale, politica, ma anche economica, in grado di contrastare l'imposizione democratica dell'individualismo liberale. Fu questa una vera e propria "teologia politica" del XX secolo, la cui premessa fondamentale consistette - almeno secondo l'ideatore di questa teoria - nel «cambiare qualcosa affinché tutto rimanesse uguale» (cambiar algo para que todo siga igual). Nonostante ciò, il cambiamento sociale e politico della Spagna non avvenne sulla base della proposta tecnocratica di questi burocrati del vecchio Stato franchista, ma a partire dalla "nuova rotta democratica" che caratterizzò l'epoca conosciuta oggi come Transizione politica spagnola. 


\section{* Gli autori}

Antonio César Moreno Cantano ha ottenuto il titolo di Dottore in Storia contemporanea presso l’Universidad de Alcalá de Henares (2008). Ha preso parte a diverse conferenze nazionali e internazionali sul tema della dittatura franchista e ha pubblicato, su differenti riviste specializzate, numerosi articoli sulla propaganda interna ed estera della Spagna franchista durante la Guerra civile e la Seconda guerra mondiale. Ha coordinato, presso la casa editrice Trea una trilogia sulla cultura bellica e la propaganda in Spagna tra il 1936 e il 1945 (El ocaso de la verdad: propaganda y prensa exterior en la España franquista (1936-1945), Gijón, Trea, 2011; Propagandistas y diplomáticos al servicio de Franco, 1936-1945, Gijón, Trea, 2012; Cruzados de Franco, Gijón, Trea, 2013). La sua ultima pubblicazione è inserita nell'opera collettiva Otra Iglesia. Clero disidente durante la Segunda República y la Guerra Civil (Gijón, Trea, 2014).

URL: < http://www.studistorici.com/2015/o9/24/antonio-cesar_moreno-cantano/ >

Matteo Tomasoni ha conseguito il titolo di dottore di ricerca in Storia presso l'Universidad de Valladolid (Spagna, 2014), con una tesi sul fascismo spagnolo. Già dottore magistrale in Storia d'Europa presso l'Università di Bologna (2008), negli ultimi anni ha svolto attività di ricerca tra Spagna, Italia e Germania e collabora con vari gruppi fra cui il SIdIF (Seminario Interuniversitario de Investigadores del Fascismo), e la rivista «Zibaldone. Estudios italianos» di cui è membro della redazione. I suoi interessi sono rivolti allo studio dell'evoluzione storica del fascismo e dei movimenti politici del periodo tra le due guerre mondiali, oltre allo studio di alcuni aspetti della Prima guerra mondiale.

URL: < http://www.studistorici.com/2008/o9/14/matteo-tomasoni/ >

\section{Per citare questo articolo:}

MORENO CANTANO, Antonio César, TOMASONI, Matteo, «Panoramica: Spagna 2015», Diacronie. Studi di Storia Contemporanea : La voce del silenzio: intelligence, spionaggio e conflitto nel XX secolo, 29/12/2016,

URL:< http://www.studistorici.com/2016/12/29/spagna_numero_28/ >

Diacronie Studi di Storia Contemporanea $\vartheta$ www.diacronie.it

Risorsa digitale indipendente a carattere storiografico. Uscita trimestrale. redazione.diacronie@hotmail.it

Comitato di redazione: Jacopo Bassi - Luca Bufarale - Antonio César Moreno Cantano - Deborah Paci - Fausto Pietrancosta - Alessandro Salvador - Matteo Tomasoni - Luca Zuccolo

Diritti: gli articoli di Diacronie. Studi di Storia Contemporanea sono pubblicati sotto licenza Creative Commons 3.0. Possono essere riprodotti e modificati a patto di indicare eventuali modifiche dei contenuti, di riconoscere la paternità dell'opera e di condividerla allo stesso modo. La citazione di estratti è comunque sempre autorizzata, nei limiti previsti dalla legge. 